\title{
Diseño de un Sistema Integral de Análisis del Turismo (SIAT) en destinos patrimoniales
}

\author{
Yamilé Pérez Guilarte \\ Universidad de Santiago de Compostela \\ yamile.perez@rai.usc.es
}

\section{RESUMEN}

Las ciudades declaradas Patrimonio de la Humanidad se enfrentan al reto de conservar su patrimonio, gestionar altos flujos de visitantes, y al mismo tiempo ofrecer una experiencia acorde a la demanda actual. El objetivo de este trabajo es diseñar una metodología para la implementación de un Sistema Integral de Análisis del Turismo (SIAT) en ciudades patrimoniales. Se parte de la elaboración de una guía dirigida a estudiar los observatorios turísticos, herramienta más utilizada en los destinos patrimoniales. Se establecieron como casos de estudio los observatorios turísticos de cinco ciudades españolas: Córdoba, Ávila, Segovia, Salamanca y Santiago de Compostela. Los resultados demuestran que los observatorios turísticos realizan un seguimiento de los principales indicadores de la demanda y la oferta. Sin embargo, hay aspectos menos abordados relacionados con el impacto económico, la población local o el uso de las TIC en la recopilación y difusión de la información. EI SIAT se fundamenta sobre la base de dos pilares. Por una parte, propone un Subsistema de Recopilación y Análisis de Datos, dirigido a mejorar la competitividad del destino. Por otra, plantea un Subsistema de Investigación para el desarrollo de proyectos orientados a velar por la sostenibilidad turística.

Palabras clave: Patrimonio de la Humanidad, Competitividad, Sostenibilidad, Observatorios Turísticos. 


\title{
Implementation of an Integral System for Tourism Analysis (ISTA) in heritage cities
}

\author{
Yamilé Pérez Guilarte \\ Universidad de Santiago de Compostela \\ yamile.perez@rai.usc.es
}

\begin{abstract}
The World Heritage cities face the challenge of preserving its heritage, managing visitor flows, while providing an experience in line with current demand. The aim of this paper is to design a methodology for the implementation of an Integral System for Tourism Analysis (ISTA) in heritage cities. The work starts by developing a study guide for tourist observatories, the most used tool in heritage destinations. The tourism observatories of five Spanish cities were established as cases of study: Córdoba, Avila, Segovia, Salamanca and Santiago de Compostela. The results show that tourism observatories keep track of the main indicators of demand and supply. However, aspects related to the economic impact of tourism, the local population or the use of ICT in the collection and dissemination of information are less exploited. The ISTA is founded based on two pillars. First, it presents a Subsystem of Collection and Analysis of information aimed at improving the competitiveness of the destination. Secondly, it suggests a Subsystem of Research for developing projects aimed at ensuring the sustainability of the tourist activity.
\end{abstract}

Keywords: World Heritage, Competitiveness, Sustainability, Tourism Observatories. 


\section{INTRODUCCIÓN}

Los elevados flujos de visitantes que acogen las ciudades patrimoniales conllevan la necesidad de prestar especial atención a la preservación de su entorno. Para evitar problemas como la banalización de la cultura y la pérdida de identidad local es esencial garantizar la calidad de vida de los residentes y la experiencia de los visitantes. Al mismo tiempo, los destinos patrimoniales se enfrentan al reto de ofrecer un producto turístico competitivo que trascienda las tradicionales visitas a monumentos, edificios y lugares históricos.

Para implementar las acciones adecuadas en materia de competitividad y sostenibilidad de los destinos patrimoniales, es necesaria la disponibilidad de herramientas que permitan un análisis integral del desarrollo de la actividad turística en el destino. El monitoreo sistemático de determinados indicadores turísticos resulta clave para la toma de decisiones. De este modo, se impone el estudio de aspectos como: la demanda turística (perfil sociodemográfico, comportamiento, preferencias, satisfacción, imagen, etc.), el comportamiento de las empresas que intervienen en el mercado turístico, el impacto del turismo en el conjunto de la economía o la satisfacción de la población local. De igual modo, deben realizarse investigaciones para gestionar los flujos de visitantes, profundizar en el comportamiento de productos turísticos específicos o hacer uso de las tecnologías de la información y la comunicación en beneficio de la investigación turística.

El objetivo de este trabajo es diseñar una metodología para crear un Sistema Integral de Análisis del Turismo (SIAT) en ciudades Patrimonio de la Humanidad. Los observatorios turísticos son un instrumento ampliamente utilizado en destinos patrimoniales. Por tanto, se parte de una guía para evaluar la adecuación de los mismos a las actuales necesidades de información. Considerando la experiencia de los observatorios turísticos en España, se seleccionaron cinco ciudades españolas como casos de estudio: Córdoba, Ávila, Segovia, Salamanca y Santiago de Compostela.

\section{ANTECEDENTES}

La Organización Mundial de Turismo siempre ha manifestado la necesidad de la creación de un Sistema de Estadísticas de Turismo (SET), que permitiera integrar los Sistemas Generales de Estadísticas. De este modo, se puso en marcha la Cuenta Satélite de Turismo que proporcionaría información fiable, consistente y apropiada sobre la estructura socioeconómica y el desarrollo del turismo (Naciones Unidas, 2010). Sin embargo, en muchos casos los sistemas estadísticos no responden a todas las necesidades de los diferentes agentes que integran el turismo. Cada usuario requiere un tipo de información específica y adaptada a su actividad, lo que hace necesario un planteamiento más integral como son los Sistemas de Información Turística (SIT) (Valdés, Del Valle y Sustacha, 2011).

Bigné, Font y Andreu (2000) definen el Sistema de Información Turística como aquel proceso permanente y sistematizado de recopilación, tratamiento, ordenación y distribución de la información, utilizada por agentes públicos y privados para la planificación, acción y evaluación de un destino. De este modo, los SIT permiten evaluar las posibilidades de captación de un mayor flujo de turistas; segmentar los mercados; detectar situaciones que 
necesiten una investigación específica, conocer la opinión de los clientes sobre el destino o estudiar a los competidores. En fin esta herramienta proporciona una visión global de la situación turística del destino, que permite a los agentes turísticos una acertada planificación y gestión del mismo.

Desde el punto de vista organizativo, en algunos países los Sistemas de Información Turística han formado parte de los trabajos de las organizaciones de mercadotecnia o gestión de destinos (DMO). Mientras que, en otros destinos y regiones se ha considerado la necesidad de recopilar datos e informaciones de sus visitantes de forma sistemática y estructurarlos en análisis y publicaciones asequibles a los actores del turismo, utilizando para ello los Ilamados Observatorios Turísticos (Valdés, Del Valle y Sustacha, 2011). Estas entidades, con variadas subordinaciones y fuentes de financiación, profundizan en el conocimiento del fenómeno turístico, especialmente en el comportamiento de la demanda, aunque también analizan la oferta turística.

El análisis del fenómeno turístico es el punto en común de todos estos sistemas, sin embargo, existen algunas diferencias según las bases de cada uno. En España, por ejemplo, son pocos los observatorios que generan información primaria, sobre todo de la demanda. Por el contrario, hay muchas iniciativas vinculadas con la difusión de las estadísticas de los registros administrativos, principalmente en lo referente a la oferta de establecimientos turísticos (García, 2009). A este respecto, Massieu (2000) establece que todo sistema de información turística debe incluir en su metodología de trabajo los siguientes aspectos:

- Conceptos básicos sobre la estadística turística,

- Clasificaciones de productos y actividades turísticas,

- Metodología de recopilación de información (encuestas, registros administrativos, directorios, censos, etc.),

- Almacenamiento (bases de datos),

- Metodología de tratamiento de la información (análisis y proceso de datos),

- Difusión de la información (página web, informes, publicaciones específicas).

En España uno de los ejemplos destacados es el Sistema de Información Turística de Asturias (SITA), el cual constituye un sistema integral para el estudio y análisis del turismo en un espacio socioeconómico. El SITA realiza el trabajo a través de una serie de líneas de investigación organizadas en cuatro bloques básicos de información, perfectamente complementarios y sinérgicos. Ellos son: el análisis de la demanda (en destino y origen), el estudio de la oferta, la estimación del impacto económico que genera la actividad turística, así como un área de investigación y conocimiento (Valdés, Martín y Aza, 2011).

Los observatorios turísticos revisten una especial importancia en el caso de las ciudades patrimoniales, aunque una gran parte de ellas aún no han apostado por este tipo de herramientas. La Organización de las Ciudades del Patrimonio Mundial (OCPM) reúne 238 ciudades cuyos centros históricos, monumentos o zonas han sido declarados Patrimonio de la Humanidad. Estas ciudades se concentran fundamentalmente en Europa (Figura 1). En la región de Latinoamérica y el Caribe, México encabeza el ranking de las ciudades patrimoniales con 11, seguido de Brasil con 7 y Cuba con 4 . Hay que destacar que países 
como Estados Unidos, Argentina o la India, este último con una gran cantidad de sitios declarados Patrimonio Cultural (23), no cuentan con ninguna ciudad (OCPM, 2013).

Figura 1. Distribución de las ciudades pertenecientes a la Organización de Ciudades del Patrimonio Mundial.

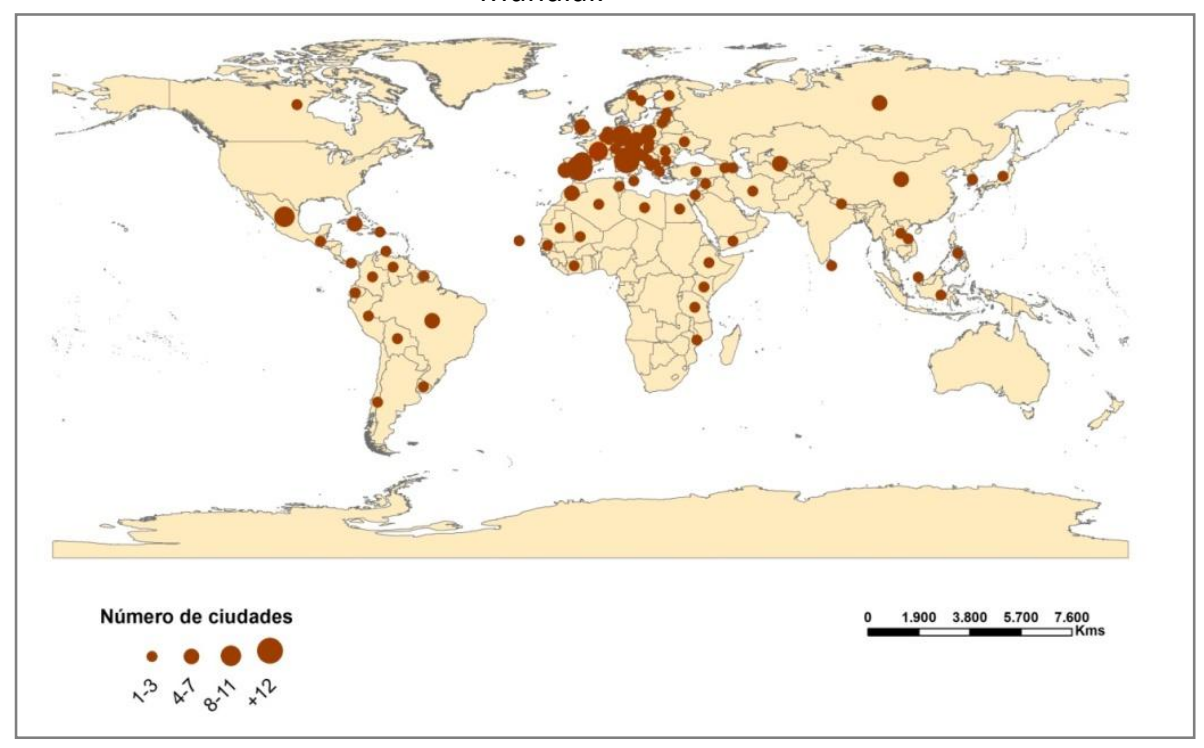

Fuente: Organización de Ciudades del Patrimonio Mundial (OCPM, 2013).

En algunos destinos como España sí que se aprecia una correspondencia entre el número de ciudades Patrimonio de la Humanidad y el de observatorios turísticos. De este modo, algunas de las ciudades patrimoniales españolas (Figura 2) han creado observatorios turísticos, entras ellas: Córdoba, Ávila, Tarragona, Cuenca, Santiago de Compostela, Ibiza, Salamanca, Toledo y Segovia. A su vez, el Grupo de Ciudades Patrimonio de la Humanidad de España (GCPHE) creó un observatorio conjunto. El mismo elabora y publica anualmente un boletín de coyuntura turística, en el que se analizan los principales indicadores turísticos de las ciudades en conjunto, y de forma individual (Troitiño et al., 2009).

Figura 2. Ciudades Patrimonio de la Humanidad de España

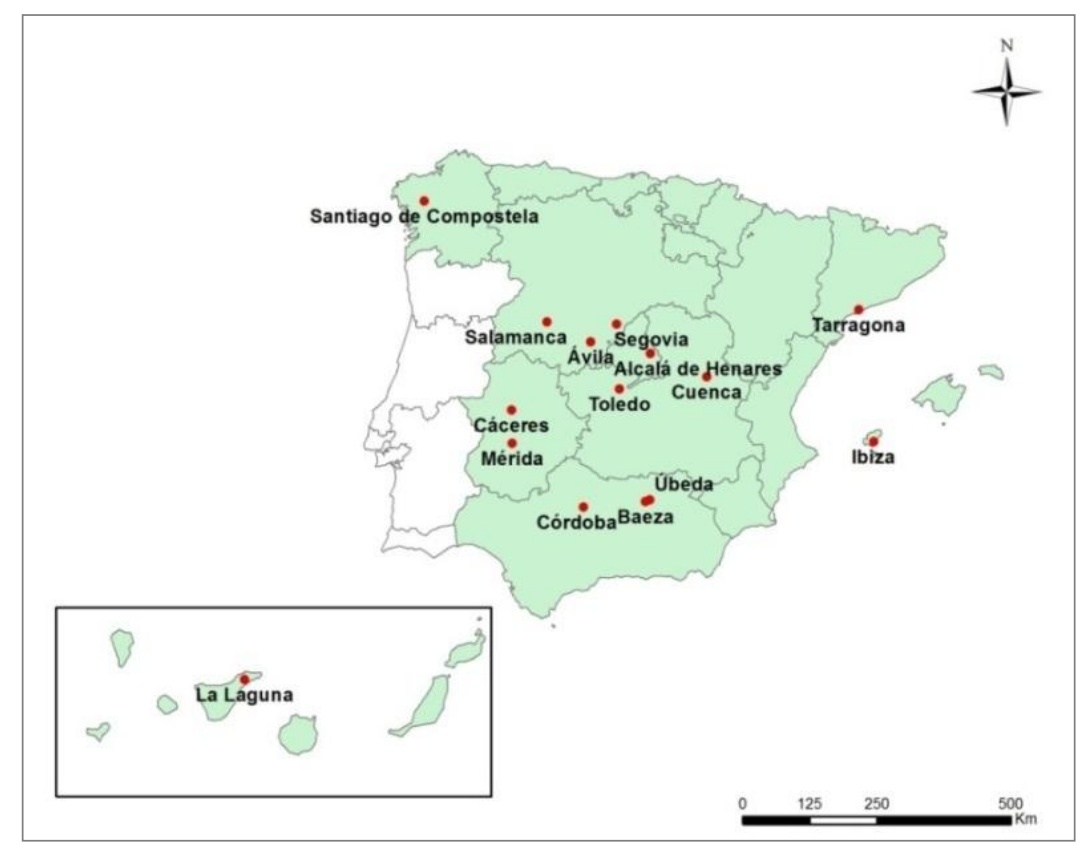

Fuente: García Nieto (2014). 
La situación que existe en España en cuanto al desarrollo de observatorios turísticos no se aprecia de igual manera en otras regiones como es el caso de Latinoamérica, por poner un ejemplo. A pesar del gran número de ciudades Patrimonio de la Humanidad en esta región (Figura 1), no se puede decir lo mismo de las que poseen observatorios turísticos. No obstante, destacan iniciativas en México, como el Observatorio de la Asociación de Ciudades Mexicanas del Patrimonio Mundial (ANCMPM, 2015) o los Observatorios Turísticos de los Estados de Guanajuato y Puebla (SECTUR, 2015; Rodríguez et al., 2013). Otro ejemplo es el Observatorio de Colonia de Sacramento (Uruguay) y el Sistema de Información Turística de La Habana (SITCHA). Este último fue creado en 2005 con la colaboración del Sistema de Información Turística de Asturias (Váldes, Martín y Aza, 2011), pero no pudo continuar su labor por problemas de financiamiento (Pérez Guilarte, 2014). La falta de implementación de este tipo de herramientas representa una importante desventaja competitiva para cualquier destino, y en especial para los patrimoniales.

\section{METODOLOGÍA DE INVESTIGACIÓN}

De los antecedentes se desprende que los observatorios turísticos son la herramienta más utilizada en ciudades patrimoniales, pero también que estos están orientados básicamente al estudio de la demanda y la oferta. Este trabajo propone una metodología para implementar un Sistema Integral de Análisis del Turismo (SIAT). Se partió del diseño de una guía (Anexo) para conocer la adecuación de un observatorio turístico a las necesidades actuales en materia de investigación en un destino patrimonial. La guía, además del estudio de la oferta y la demanda, recoge aspectos como: el impacto económico, la opinión de la población local, la investigación dirigida a la organización de los flujos turísticos y a la estimación de los límites de acogida. A su vez, incluye el tema del trabajo en red con otros observatorios y el uso de las tecnologías de la información y la comunicación en la obtención, y particularmente en la difusión de los datos generados.

Para la elaboración de la guía se consultó la literatura publicada y se revisaron ejemplos de sistemas existentes tanto a nivel nacional, como regional, provincial o municipal. De este modo, se estudió la metodología seguida por el Sistema de Información Turística de Asturias (SITA) (Valdés, Martín y Aza, 2011), el Sistema de Análisis y Estadística del Turismo de Andalucía (SAETA) (Aurioles-Martín, 2000; Gallego et al., 2001), el Sistema de Información y Estadística Turística de Argentina (SIET, 2015) o los ya mencionados observatorios creados por las asociaciones de ciudades patrimoniales en España y México. Estos sistemas han sido denominados utilizando frases como "Sistema de Información", como es el caso del SITA o el SIET, que pudieran ser confundidos con sistemas de información al visitante. Por este motivo, este trabajo propone la terminología Sistema Integral de Análisis del Turismo (SIAT) para hacer referencia a los sistemas que cumplan con la metodología que se propone.

La guía fue utilizada para estudiar los observatorios turísticos de cinco ciudades patrimoniales españolas. Se seleccionaron tres ciudades consolidadas como Santiago de Compostela, Córdoba y Salamanca; así como los casos de Ávila y Segovia, ciudades patrimoniales emergentes (Troitiño y al., 2009). La metodología que se siguió fue la utilización de la información publicada en sus respectivas páginas webs. En el caso de 
Santiago, debido a la poca información disponible en su web, se realizó una entrevista al director del observatorio turístico.

\section{RESULTADOS}

En este apartado se exponen los resultados de la revisión de las páginas webs de los observatorios turísticos de Córdoba, Ávila, Segovia, Salamanca y Santiago de Compostela. En este último caso, se incorporan también las consideraciones obtenidas a partir de la entrevista efectuada al director del observatorio. La presentación de los resultados se divide en los tres apartados que estructuran la guía (Anexo): Datos generales; Recopilación, procesamiento y difusión de la información; e Investigación, redes de cooperación y TIC. De este modo, se integran los resultados de los cinco casos de estudio y se establece una comparativa entre ellos. Por último, se presenta la metodología propuesta para implementar un Sistema Integral de Análisis del Turismo (SIAT) en ciudades patrimoniales.

\subsection{Datos Generales}

A continuación se describen los observatorios que fueron seleccionados para este estudio. Como muestra la Tabla 1 se presentan algunos aspectos como: la fecha de creación, las entidades que lo coordinan y la página web en la que se difunde sus contenidos. El Observatorio Turístico de la Ciudad de Córdoba, se puso en marcha en la segunda mitad del año 2001, como una solución a la falta de disponibilidad de información turística en la ciudad. Se trata de una iniciativa del Ayuntamiento de Córdoba, en colaboración con la Caja General de Ahorros de Granada, a través de la Sociedad de Estudios Económicos de Andalucía (ESECA), y con la participación del sector turístico. Desde el año 2003 el observatorio es coordinado por el Consorcio de Turismo de Córdoba, disponiendo de una sección dentro de su página web oficial (Turismo de Córdoba, 2014).

Tabla 1. Información de los observatorios turísticos en estudio.

\begin{tabular}{|c|c|c|c|}
\hline Observatorio & Año & Entidades & Página web \\
\hline Córdoba & 2001 & $\begin{array}{c}\text { Consorcio de Turismo de } \\
\text { Córdoba }\end{array}$ & $\frac{\text { www.turismodecordoba.org/ob }}{\frac{\text { servatorio-turistico.cfm }}{\text { Ávila }}}$ \\
\hline Segovia & 2002 & $\begin{array}{c}\text { Ayuntamiento de Ávila y } \\
\text { Universidad Complutense de } \\
\text { Madrid }\end{array}$ & $\frac{\text { www.avilaturismo.com/es/area }}{\text {-profesional/boletin-tco }}$ \\
\hline Salamanca & 2006 & $\begin{array}{c}\text { Ayuntamiento de Segovia y } \\
\text { Universidad Complutense de } \\
\text { Madrid }\end{array}$ & $\frac{\text { www.turismodesegovia.com/es }}{\text { Aprensa/observatorio-turistico }}$ \\
\hline $\begin{array}{c}\text { Santiago de } \\
\text { Compostela }\end{array}$ & 2005 & $\begin{array}{c}\text { Ayuntamiento de Salamanca } \\
\text { Santiago }\end{array}$ & $\frac{\text { www.salamanca.es/es/profesio }}{\text { nales/item/210-observatorio- }}$ \\
\hline
\end{tabular}

Fuente: Turismo de Córdoba (2014), Ayuntamiento de Ávila (2014), Turismo de Segovia (2014), Turismo de Salamanca (2014) y Observatorio de Santiago de Compostela (2014). 
Por su parte, el Observatorio Turístico de la Ciudad de Ávila comenzó en 2002 y actualmente funciona mediante un convenio entre el Ayuntamiento de Ávila y la Universidad Complutense de Madrid (Grupo de Investigación "Turismo, Patrimonio y Desarrollo"). Sus principales cometidos son: la creación y mantenimiento de una base de datos; el análisis periódico de la información, y su comparación con análisis previos; el desarrollo de investigaciones específicas sobre eventos que generan turismo; y la redacción del Boletín Informativo del Observatorio Turístico. Su información aparece en el área profesional de la web de turismo del ayuntamiento (Ayuntamiento de Ávila, 2014).

Segovia cuenta con un Observatorio Turístico propio desde 2008, fecha en la que se firmó un convenio entre el Ayuntamiento y la Universidad Complutense de Madrid. El Grupo de Investigación "Turismo, Patrimonio y Desarrollo" fue responsable de su montaje y puesta en marcha, pero a partir de 2010 es mantenido por la empresa municipal de turismo. El objetivo del observatorio es la recolección, el tratamiento y el análisis de la información de coyuntura turística relacionada con la evolución del turismo en la ciudad. El acceso a la información online se realiza desde el portal oficial de turismo (Turismo de Segovia, 2014).

Salamanca, al igual que el resto de ciudades patrimoniales, apuesta por el conocimiento y la investigación turística utilizando para ello un observatorio turístico creado en 2006. Su objetivo es proporcionar una herramienta para gestionar la información científica sobre el turismo en la ciudad y posibilitar un eje de análisis a los organismos públicos y privados del sector. La información que genera es accesible desde el área de profesionales del portal de turismo de Salamanca (Turismo de Salamanca, 2014).

El Observatorio Turístico de Santiago pertenece al Centro de Estudios e Investigaciones Turísticas (CETUR), que es uno de los Centros Propios de la Universidad de Santiago de Compostela. Fue creado en 2005 por encargo de Turismo de Santiago con el objetivo de establecer un mecanismo de estudio y análisis sistemático del sector turístico en la ciudad. El observatorio está integrado por agentes públicos y privados. Entre los primeros se encuentran: la Universidad de Santiago, la Xunta de Galicia y el Ayuntamiento de Santiago. Por su parte, el sector privado está representado por: la Cámara de Comercio de Santiago y la Asociación de Empresarios de Hostelería y Restauración de Santiago y comarca. El observatorio cuenta con un espacio en la página web de la Universidad de Santiago, aunque la información no se encuentra actualizada (Observatorio de Santiago de Compostela, 2014).

\subsection{Recopilación, procesamiento y difusión de información}

La recopilación y difusión de información son las actividades centrales de un observatorio turístico, pero según el caso se utilizan fuentes oficiales, institucionales, públicas, privadas o propias. El estudio cualitativo de los visitantes constituye un elemento prácticamente de obligatoriedad en un observatorio local, pues es desde este ámbito que puede obtenerse información más fiable. Al respecto, los Observatorios de Ávila y Segovia utilizan la información de las oficinas o centros de atención al visitante. Mientras que, los Observatorios de Córdoba, Salamanca y Santiago optan por realizar encuestas a los visitantes. El hecho de entrevistar a las personas permite recopilar un volumen mayor de información, tanto del perfil como del comportamiento, el grado de satisfacción y la imagen del destino. La metodología seguida por estos tres observatorios es similar, estimando un tamaño de muestra anual por encima de los 1.000 visitantes. El Observatorio de Santiago 
entrevista el mayor número de visitantes (1.842), a continuación el de Córdoba (1.600) y por último el de Salamanca (1.038 en 2011).

Para realizar los análisis de la evolución del número de viajeros y las pernoctaciones, los observatorios de Segovia, Ávila y Córdoba utilizan los datos procedentes del Instituto Nacional de Estadística (Encuesta de Ocupación Hotelera). En el caso de Salamanca, los boletines disponibles en su web sólo hacen referencia al perfil y comportamiento de los visitantes. Tampoco desde el Observatorio de Santiago se realiza un seguimiento de estos indicadores cuantitativos.

Con relación a la oferta, se lleva a cabo el estudio del comportamiento del número de establecimientos y plazas de alojamiento a través de la utilización de fuentes secundarias autonómicas. En el caso de Ávila, del registro de establecimientos turísticos de la Junta de Castilla y León; y en el caso de Córdoba del Sistema de Información Multiterritorial de Andalucía (SIMA). Por su parte, el Observatorio de Santiago ha realizado algunos estudios dirigidos fundamentalmente a hoteles y restaurantes para comprobar la situación de algunas variables relacionadas con el empleo, la formación, las inversiones, la comercialización o la calidad del establecimiento.

También han sido objeto de estudio del Observatorio de Santiago productos específicos como el turismo de congresos, el turismo cultural, el gastronómico, el idiomático o el residencial. Otra línea de investigación desarrollada fue el estudio del perfil del peregrino, para lo cual se creó a finales de 2006 el Observatorio del Camino de Santiago, aunque dejó de funcionar en 2010 por falta de financiamiento. De igual modo, el observatorio cuenta con una encuesta para conocer el grado de conocimiento que tiene la población local de la política turística, su valoración y su identificación con la ciudad turística. Sin embargo, el director del observatorio señaló que todos estos estudios no se estaban efectuando como consecuencia de la falta de presupuesto.

El Observatorio de Santiago ha participado en la recopilación de información para varios proyectos de investigación. De este modo, entre marzo de 2008 y abril de 2009 se realizó un estudio para conocer la capacidad de acogida y la ordenación de flujos en la Catedral de Santiago. Este proyecto fue financiado por la Dirección General de Turismo de la Xunta de Galicia y en el cual colaboró el Grupo "Turismo, Patrimonio y Desarrollo" de la Universidad Complutense de Madrid. De igual modo, formó parte del proyecto ATLANTE que surgió de un acuerdo transnacional firmado en 2003 entre los municipios atlánticos gallegos y portugueses con centros históricos declarados Patrimonio de la Humanidad, en el ámbito del Fondo Europeo de Desarrollo Regional (FEDER). Asimismo, se realizaron encuestas a los visitantes para determinar su perfil como parte del proyecto ATLAS (http://www.tramresearch.com/atlas/presentation.htm) del año 2004.

Con relación a la difusión, hay que destacar la elaboración de informes que divulgan la información generada. El Observatorio de Córdoba publica boletines de carácter trimestral y un informe anual a través de su página web. Este último propone la detección de las fortalezas, oportunidades, amenazas y debilidades de la ciudad como destino turístico, a través de un análisis tanto de la oferta como de la demanda. En el caso del Observatorio de Segovia los informes son publicados en su web de forma anual o bianual, mostrando un análisis integral del sector turístico. Además del estudio de los visitantes y la ocupación 
hotelera, se realiza un seguimiento de las oficinas de turismo, los principales monumentos y museos de la ciudad, los productos turísticos de mayor atracción, las webs de turismo y las redes sociales. Hay que destacar que también se llevan a cabo encuestas a los visitantes a través de su web.

El Observatorio de Ávila publica un balance anual de la actividad turística, un boletín correspondiente al primer semestre y otro de la actividad turística durante el verano. Además de los datos de coyuntura turística, se han abordado las visitas en grupos organizados, la situación en comparación con las ciudades del Grupo de Ciudades Patrimonio de la Humanidad de España, o el impacto del destino en las redes sociales. Asimismo, se han estudiado temas como el éxito de la peatonalización de la Muralla con fines turísticos, el impacto de la feria gastronómica "Ávila en tapas", o el peso de la oferta de los cursos de español para extranjeros en la ciudad.

Por su parte, el Observatorio de Salamanca publica también a través de su web los boletines anuales correspondientes al estudio de los visitantes, aunque el último se remonta a 2011. Además, se realizan estudios de productos específicos como el turismo de reuniones y congresos, y el idiomático. En el caso del Observatorio de Santiago desde 2007 no se publica en su web ningún resultado de la demanda. Tampoco son publicados de forma digital o impresa los boletines que elabora el observatorio, ya que el Ayuntamiento de Santiago es el único destinatario de la información.

Además de la información generada por el propio observatorio, los boletines emitidos también son un medio de difusión de resultados de proyectos de investigación o eventos científicos realizados por las instituciones que los financian o promueven. Por ejemplo, el Observatorio de Ávila publicó los resultados del estudio de la afluencia de visitantes, la rehabilitación y el proceso de puesta en valor turístico de la Muralla de Ávila (boletín №2, octubre de 2002). De igual modo, el Boletín № 1 de mayo de 2002 recoge las conclusiones del "II Encuentro de Modelos de Gestión Turística en las Ciudades Patrimoniales: Modelos para la Gestión Turística del Patrimonio" y las "III Jornadas de Turismo Cultural Ciudad de Ávila".

\subsection{Redes de cooperación y uso de las TIC}

El hecho de que las ciudades en estudio formen parte del Grupo de Ciudades Patrimonio de la Humanidad de España garantiza la realización de acciones de colaboración. Además de la promoción conjunta de estos destinos, se desarrollan proyectos y propuestas que a partir de problemáticas en común permiten el intercambio de experiencias. Asimismo, el Observatorio Turístico del Grupo ha facilitado el análisis de la situación de la actividad turística en las ciudades que lo integran.

Con respecto a la explotación de las TIC destaca el caso de Segovia. En su informe se incluye el análisis del impacto de las páginas webs y los perfiles en redes sociales del destino. De este modo, se contabiliza el número de visitas a las páginas, y la cantidad de amigos y seguidores en Facebook o Twitter, y los usuarios de la Tarjeta Turística de la ciudad. A la par, se realizan encuestas a través de la web de Turismo de Segovia para conocer su satisfacción y también su opinión en temas concretos. En este último caso se encontraron preguntas como por ejemplo: ¿qué recuerdo te llevarías de Segovia? 
El Observatorio de Ávila también publica información del impacto de Turismo de Ávila en las redes sociales. Por ejemplo, se realiza un seguimiento a los seguidores en Facebook y Twitter, no sólo en cuanto al número, sino también a los que comentan y comparten las publicaciones o su perfil (edad, sexo, procedencia, etc.). En el caso de los observatorios de Salamanca y Córdoba, sí que hacen uso de Internet para publicar la información generada, aunque no hay referencias en los boletines de un uso más amplio de las TIC para recopilar datos de los visitantes. El observatorio de Santiago sólo obtiene información de las encuestas realizadas personalmente a los visitantes.

\subsection{Diseño de un Sistema Integral de Análisis del Turismo (SIAT) en ciudades patrimoniales.}

Siguiendo los parámetros recogidos en la guía diseñada y a partir de los resultados obtenidos por los observatorios estudiados, se propone un Sistema Integral de Análisis del Turismo (SIAT) para ser implementado en ciudades patrimoniales. Éste se basa en la integración de dos grandes subsistemas (Figura 3). Por una parte, el Subsistema de Recopilación y Análisis de Datos, un mecanismo continuo que constituya un barómetro de la actividad turística, y en consecuencia, una herramienta de análisis para todos los actores que intervienen en el sector. Por otra, el Subsistema de Investigación, orientado al desarrollo de proyectos que garanticen la protección del patrimonio material e inmaterial, vital para la sostenibilidad a largo plazo de este tipo de destinos. 
Figura 3. Metodología de un Sistema Integral de Análisis del Turismo (SIAT)

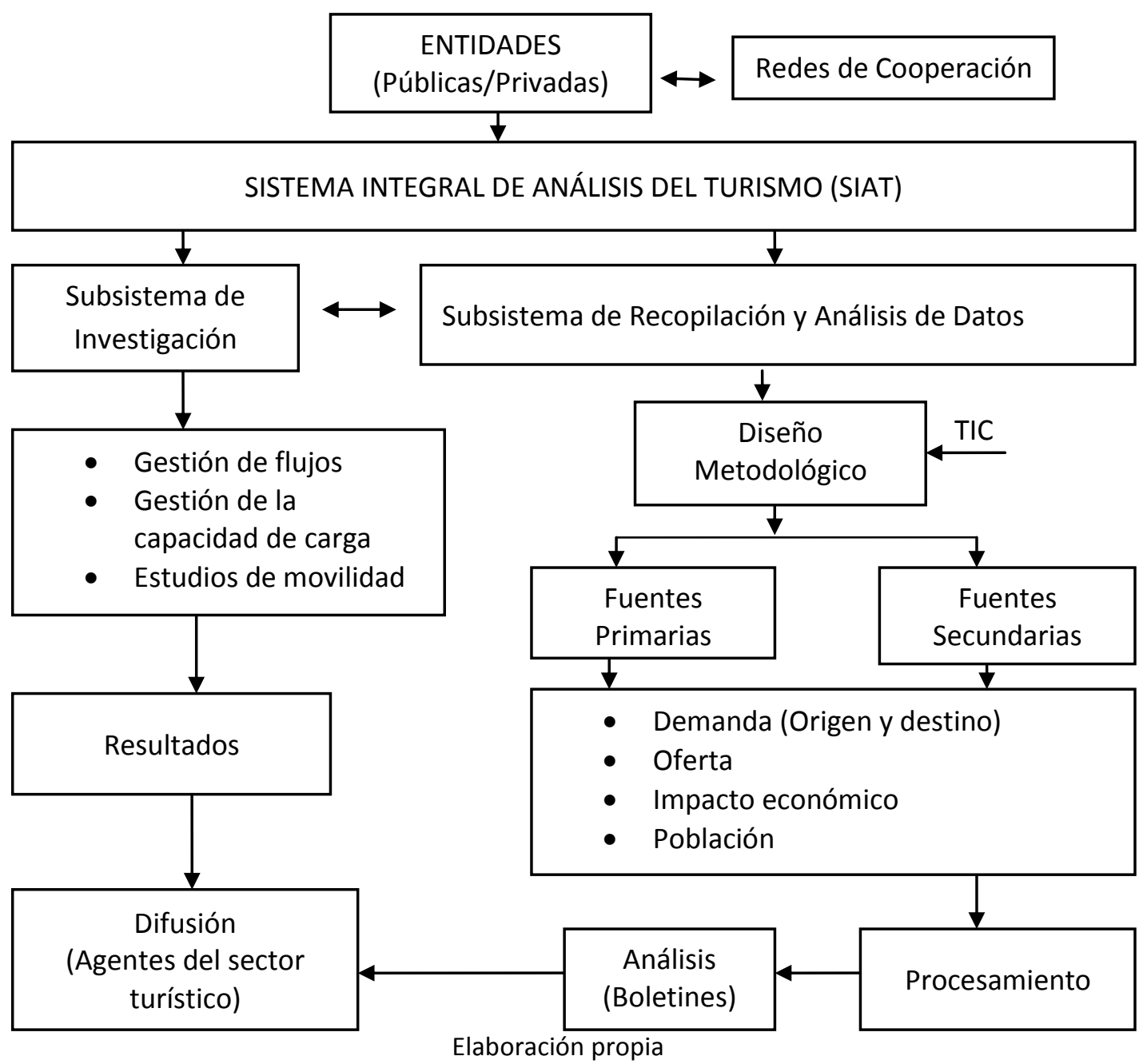

Como muestra la Figura 3, se propone que el SIAT esté integrado por entidades tanto del sector público como del privado. De este modo, se lograría una colaboración entre todos los agentes que intervienen en el sector turístico, y en consecuencia, se garantizaría un uso eficiente de las potencialidades que brinda este tipo de sistemas. En el caso del sector público, es vital el liderazgo de la entidad que tiene la competencia en la planificación y gestión turística, dígase por ejemplo Turismo de Santiago o Turismo de Segovia. Asimismo, como se pudo apreciar en algunos de los observatorios estudiados, como el de Ávila, Segovia y Santiago de Compostela, la universidad desempeña un papel fundamental en el desarrollo de un observatorio. En el caso del SIAT, también se propone el trabajo con académicos y grupos de investigación con experiencia en este ámbito y con capacidad de trabajo en redes de cooperación. Por su parte, las empresas deben estar representadas a través de asociaciones de hostelería, de restauración, de agencias de viajes, de comerciantes, etc.

El Subsistema de Recopilación y Análisis de Datos está concebido para el análisis sistemático de la demanda en destino y origen, la oferta, el impacto económico que genera la actividad, y la percepción de la población con relación al turismo. A continuación será detallada la metodología propuesta para el estudio de cada uno de estos parámetros. Para ello, se comentarán las variables establecidas para su análisis, las fuentes y técnicas para la recopilación de información y su procesamiento, así como los mecanismos de difusión. 
En el caso de la demanda, se considera necesaria la recopilación de datos primarios a través de la realización de encuestas a los visitantes. Estos deberán ser seleccionados de forma aleatoria en los principales puntos turísticos de la ciudad, el aeropuerto o las estaciones de autobuses o ferroviarias. El trabajo de recogida se deberá distribuir a lo largo del año, estimando el volumen de visitantes a entrevistar según los flujos turísticos en cada época, y a partir de la fijación de un nivel de confianza de al menos un 95\% y un error menor de un $5 \%$. En el diseño del cuestionario se tendrá en cuenta la incorporación de variables como: la motivación y forma de organización del viaje, las fuentes de información, las actividades de interés, el gasto desagregado por servicios, la satisfacción e imagen del destino, así como el perfil sociodemográfico. Un aspecto a considerar para el trabajo de campo es la introducción de dispositivos como tabletas que agilicen la recogida de información, faciliten el control del trabajo y su posterior procesamiento. De igual modo, es recomendable la obtención de información sobre la imagen del destino a través del seguimiento de su perfil en las redes sociales, de blogs de viajes como Tripadvisor, o de cuestionarios online.

Los estudios de la demanda en origen implican un coste elevado, lo cual ocasiona que en muchos casos no se realicen este tipo de trabajo, impidiendo obtener información relevante sobre los visitantes potenciales. En este sentido, podría utilizarse como alternativa los convenios de colaboración entre universidades. De este modo, se podrían emplear redes ya existentes entre grupos de investigación de universidades de diferentes países. Por ejemplo, la Universidad de Burdeos podría hacer un estudio a los franceses sobre la imagen que tienen de Santiago de Compostela como destino turístico; y viceversa, la Universidad de Santiago, evaluar la imagen de los españoles sobre Burdeos.

De forma complementaria a la información de la demanda recogida a partir de las encuestas a los visitantes, podría realizarse un monitoreo del número de viajeros y pernoctaciones a partir de la Encuesta de Ocupación Hotelera del INE. Asimismo, sería valioso contar con un mecanismo de seguimiento de las visitas realizadas a los principales hitos monumentales y museos del destino, así como del consumo de los principales productos turísticos (visitas guiadas, alquiler de audioguías o uso del autobús o tren turístico). Este monitoreo sería posible a partir del control de ventas de entradas y de productos en cada establecimiento, sin embargo la transferencia de esta información al SIAT requeriría una estrecha colaboración con estas instituciones. Asimismo, debe fomentarse el estudio de productos específicos como el turismo de congresos o el educativo; así como o el impacto de ferias, festivales u otros eventos que sean relevantes para el destino.

Con relación a la oferta deben ser monitoreados indicadores como: la evolución del número de alojamientos y plazas por tipo de alojamiento. Estos datos pueden ser obtenidos a partir de los registros ya existentes en las fuentes de información municipales o regionales. Además, se pueden complementar con la realización de una encuesta de periodicidad anual a gerentes, encargados y responsables de establecimientos turísticos (hoteles, restaurantes, agencias de viajes, etc.) para conocer la situación de variables más específicas. El cuestionario podría incluir información sobre parámetros como: el nivel de formación de los empleados, las inversiones realizadas, las acciones de comercialización y promoción, el nivel de equipamiento tecnológico, los precios, las políticas medioambientales y las certificaciones de calidad. El tamaño de muestra debe estar condicionado por el número total de 
establecimientos turísticos por categoría. De este modo, de ser posible se entrevistarán todos los existentes; de lo contrario, se seleccionará un conjunto que sea representativo.

Para el estudio del impacto en destinos patrimoniales se propone la utilización del modelo input-output. Esta metodología ha sido empleada por diversos autores para el análisis de la actividad turística (Fletcher, 1994; Archer y Fletcher1996, Frechtling 1999; Crompton et al. 2001; Tyrrell y Johnston, 2001; Tohmo, 2005; Kashiany Pfeifer-Luckett, 2011).Este modelo presenta limitaciones que podrían conllevar a un análisis sobreestimado del impacto (Adams y Parmenter, 1992; Blake y Sinclari, 2003). Sin embargo, según Dwyer et al. (2004) en pequeñas regiones las ventajas de su uso podrían sobrepasar sus limitaciones. En el contexto español se pueden citar varios trabajos que han utilizado este modelo para la medición del impacto turístico, y que han sido realizados a nivel regional o local (Herrero et al., 2003; Valdés et al., 2010; Martínez, Pereira y Pawlowska, 2013; Villafranca y Chamorro, 2007).

Teniendo en cuenta la complejidad del turismo y sus múltiples relaciones con otras actividades económicas, son muy difíciles de cuantificar los efectos de arrastre que se generan a partir del gasto turístico inicial (Martínez et al., 2013). En el caso del SIAT se propone medir el impacto directo e indirecto del turismo sobre el destino. Los efectos directos se medirían a partir del consumo turístico que generaron los visitantes durante la estancia. Para ello, se utilizarían los datos obtenidos de las encuestas realizadas a los turistas, que como se ha mencionado incluyen el gasto en el destino. Este consumo deberá estar desagregado en los siguientes conceptos: alojamiento, transporte urbano, compras, bares y restaurantes, y actividades culturales (museos, cine, teatro, etc.). Para estimar el impacto es necesario conocer el número de visitantes, el cual podrá obtenerse a partir de los datos que proporciona la Encuesta de Ocupación Hotelera o de algún otro sistema implementado en el destino. A partir de estos gastos divididos por actividad se empleará el modelo input-output para establecer los efectos indirectos del turismo, es decir, los ocasionados por su impacto en otras actividades económicas.

La población local desempeña un papel cada vez más protagónico en el desarrollo del turismo, no sólo como anfitriones, sino también como creadores de oferta (Richards, 2012; Pérez Guilarte, 2014). Por tanto, estudiar la percepción que tiene la población residente del turismo es un aspecto relevante a ser incluido en el SIAT. Se propone la realización de un cuestionario, al menos de forma anual a personas residentes en el destino. Su objetivo sería conocer aspectos como: el grado de conocimiento que tiene la población local de la política turística, su valoración y su identificación con la ciudad turística. El número de personas a entrevistar dependerá del número de habitantes del destino, estableciendo una muestra representativa a partir de la fijación de al menos un 95\% de confianza y de un error menor de $5 \%$.

La recopilación de información primaria es seguida por una etapa de procesamiento de datos, la cual se apoya en programas estadísticos como puede ser el SPSS (Statistical Package for the Social Sciences). A diferencia de los boletines consultados donde sólo se emplea la estadística descriptiva, en el caso del SIAT se propone incorporar también la inferencial. Como se ha comentado, se parte del hecho de que los tamaños de muestra para cada estudio sean representativos y permitan, por tanto, extrapolar los resultados obtenidos 
al conjunto de la población. De este modo, se podrían establecer y estudiar relaciones entre las diferentes variables en estudio.

La difusión del conocimiento generado por el SIAT es fundamental para el cumplimiento de sus objetivos. Los boletines deberán transferirse no sólo a las entidades encargadas de la gestión turística, sino al resto de actores que integran el SIAT (universidades, asociaciones y otras entidades). Independientemente de que pueda realizarse una publicación impresa, como se ha podido constatar, la vía online parece ser la más efectiva en la sociedad actual. Se propone que el SIAT cuente con su espacio dentro de la página web de la entidad municipal encargada del turismo, como es el caso de los observatorios de Segovia, Córdoba, Salamanca o Ávila. Por último, teniendo en cuenta la opinión de los destinatarios de la información, debe establecerse un mecanismo de retroalimentación que permita realizar las correcciones necesarias en el subsistema.

De forma complementaria al Subsistema de Recopilación y Análisis de Datos se propone el Subsistema de Investigación (Figura 3). En destinos patrimoniales cobran protagonismo los estudios relacionados con los flujos turísticos; la capacidad de carga del destino y de los productos patrimoniales; así como los relativos a la movilidad (Troitiño, et al., 2003; Tresserras, 2003). De igual modo, es relevante prestar atención a la conservación del patrimonio inmaterial, el cual constituye un atractivo para muchos visitantes. Los resultados del desarrollo de estos proyectos de investigación deberán transferirse a los agentes implicados en el SIAT, y difundirse de igual modo que la información generada a través del Subsistema de Recopilación y Análisis de Datos.

Para el desarrollo del Subsistema de Investigación será necesario el apoyo de las instituciones universitarias, a través de grupos de investigación con experiencia en este tipo de proyectos. Asimismo, será valiosa su capacidad para establecer acciones de cooperación con otras organizaciones, tanto nacionales como internacionales, reforzando redes que pueden contribuir al desarrollo de acciones en beneficio mutuo, como el caso de los estudios de demanda en origen. En general, se trata de un subsistema que se encargaría de mantener la sostenibilidad turística del destino, y para ello trabajará de forma coordinada y retroalimentándose con el Subsistema de Recopilación y Análisis de Datos.

\section{CONCLUSIONES}

Los observatorios turísticos son el instrumento más utilizado como soporte de la planificación y gestión turística en las ciudades patrimoniales. Del estudio de los cinco casos expuestos en este trabajo destaca la labor de difusión de los datos generados por los observatorios. Esta voluntad se aprecia en los Observatorios de Córdoba, Segovia, Salamanca y Ávila, a través de la publicación de los boletines en sus respectivas páginas webs. Sin embargo, no ocurre así en el caso del Observatorio de Santiago de Compostela, donde no se realiza su difusión. A su vez, hay que destacar que los observatorios han participado en estudios relacionados con la sostenibilidad turística.

Sin embargo, también se aprecia que podrían realizarse mejoras de cara a reforzar la competitividad y sostenibilidad que demandan en la actualidad los destinos patrimoniales. A pesar de que el estudio de la demanda desempeña un papel importante, hay que impulsar la generación de datos primarios relacionados con la oferta, el impacto económico y la 
población local. También es imprescindible desarrollar más estudios sobre los flujos de visitantes y la capacidad de acogida de destino en su conjunto, pero también de los productos patrimoniales. Asimismo, las tecnologías de la información y la comunicación pudieran ser más aprovechadas en la recopilación y difusión del conocimiento generado.

En este sentido, el Sistema Integral de Análisis del Turismo (SIAT) propone una metodología que engloba las diferentes perspectivas a tener en consideración para realizar un análisis integral de la actividad turística, especialmente en ciudades patrimoniales. Por una parte, la actividad centrada en la recopilación y análisis de datos de forma sistemática que contribuye a mejorar la competitividad del destino. Por otra, y complementándose con la anterior, el desarrollo de proyectos de investigación orientados a mantener la sostenibilidad del mismo. A través de estas dos perspectivas, el SIAT debe lograr la integración entre los diferentes actores involucrados para lograr una acertada planificación y gestión turística del destino.

\section{REFERENCIAS BIBLIOGRÁFICAS}

Adams, P. y Parmenter, B. (1992).The medium term significance of international tourism forthe Australian economy.Camberra: Bureau of Tourism Research.

Archer, B. y Fletcher, J. (1996). The economic impact of tourism in the Seychelle.Annals of Tourism Research, 23, 32-47.

Aurioles-Martín, J. (2000). El proyecto SAETA. Un instrumento para la observación regional del turismo como actividad económica. Estudios turísticos, 144-145, 103-124.

Bigné, J. E.; Font, X. y Andreu, L. (2000). Marketing de Destinos Turísticos: Análisis y Estrategias de Desarrollo. Madrid: ESIC.

Blake, A. y Sinclair, M. T. (2003).Tourism crisis management US response to September11.Annals of Tourism Research, 30(4), 813-832.

Crompton, J., Lee, S. y Shuster, T. (2001). A guide for undertaking economic impact studies: The springfest example. Journal of Travel Research, 40(1), 79-87.

Dwyer, L., Forsyth, P. y Spurr, R. (2004). Evaluating tourism's economic effects: new and old approaches, Tourism Management, 25, 307-317.

Empresa Municipal de Turismo de Segovia (2014). Boletín Informativo de Segovia. № 7 Marzo de 2014.2 Recuperado de http://www.turismodesegovia.com/es/prensa/observatorioturistico/boletines?gid $=113$

Exceltur (2014). Impactur 2013. Estudio del impacto económico del turismo sobre la economía y el empleo de las Illes Balears. Recuperado de http://www.exceltur.org/impactur/

Fletcher, J. E. (1989). Input-Output Analysis and Tourism Impact Studies. Annals of Tourism Research, 16, 514-529.

Fletcher, J. (1994). Input-output analysis. En S. Witt y L. Moutinho (Eds.) Tourismmarketing and management handbook, 2a a edición (pp. 480-484). ReinoUnido: PrenticeHallInternational.

Frechtling, D. (1999). Estimating the multiplier effects of tourism expenditures on a localeconomy through a regional input-output model. Journal of Travel Research, 37(4), 342-332.

Gallego, I.; Molina, S.P; Moniche, A. y Muñoz, M.J. (2001).Sistema de Análisis y Estadísticas del Turismo de Andalucía. En XXVI Congreso Nacional de Estadística e Investigación 
Operativa. Úbeda 6-9 de noviembre de 2001. Recuperado de http://fama2.us.es:8080/turismo/turismonet1/economia\%20del\%20turismo/andaluc ia/sistema\%20de\%20analisis\%20y\%20estadisticas\%20del\%20turismo\%20en\%20Anda lucia.pdf

García Nieto, M.A. (2014). Apertura del Programa. Seminario de la OMT sobre colaboración público-privada: El rol del Patrimonio Mundial Material e Inmaterial en la configuración de productos turísticos innovadores. Santiago de Compostela, 6 de junio de 2014.

García, M. (2009). Los observatorios turísticos locales. Un instrumento útil para la planificación y la gestión turística en destino. En Troitiño, M.A. (Ed.) Ciudades Patrimonio de la Humanidad: patrimonio, turismo y recuperación urbana(pp. 98-113) Sevilla: Universidad Internacional de Andalucía.

Herrero, L.C.; Sanz, J.A.; Bedate, A.; Devesa, M. y Barrio M.J. Del (2003). Turismo cultural e impacto económico de Salamanca 2002, Ciudad Europea de la Cultura. Valladolid: Departamento de Economía Aplicada, Universidad de Valladolid.

Kashian, R. y Pfeifer-Luckett, R. (2011). The economic impact of organized camps. Tourism Economics, 17(4), 905-916.

Martínez, F., Pereira, X. y Pawlowska, E. (2013): El turismo académico en Galicia: otra forma de contribución de las universidades a las economías locales. Cuadernos de Turismo, $32,229-242$.

Massieu, A. (2000). Sistema de Estadísticas de Turismo. Fuentes Estadísticas, 45, 6-7.

Naciones Unidas (2010). Recomendaciones internacionales para estadísticas de turismo, 2008. Serie M No83/Rev. 1. Recuperado el 17 de septiembre de 2012, de http://unstats.un.org/unsd/publication/SeriesM/Seriesm 83rev1s.pdf

Pérez Guilarte, Y. (2014). Nuevas tendencias del turismo cultural. El papel de la investigación turística en ciudades patrimoniales: los casos de Santiago de Compostela y La Habana Vieja. (Tesis doctoral). MINERVA. Repositorio Institucional de la Universidad de Santiago de Compostela. Recuperado de http://dspace.usc.es/handle/10347/11872

Richards, G. (2012a). Tourism, Creativity and Creative Industries.En Creativity and Creative Industries in Challenging Times Conference. NHTV Breda, Noviembre. Recuperado de http://www.ipl.academia.edu/Documents/in/Creative Tourism

Rodríguez, M., Perea, J, Herrera, B. y Carrasco, V. (2013). Observatorio turístico del Estado de Puebla. En Almaraz, F., Pérez, M.A., Estelina, M. (Dir.), Ciencias Administrativas y Sociales. Handbook T-III. Congreso Interdisciplinario de Cuerpos Académicos. Guanajuato: ECORFAN, pp. 122-131.

Tohmo, T. (2005). Economic impacts of cultural events on local economies: an input-output analysis of the Kaustinen Folk Music Festival. Tourism Economics, 11(3), 431-451.

Tresserras, J. (2003). El turismo cultural en países en vías de desarrollo. Portal Iberoamericano de Gestión Cultural. Recuperado de http://www.gestioncultural.org/ficheros/1 1316769776 JJuan TC y Desarrollo.pdf

Troitiño, M.A.; De la Calle, M. y García, (2003). Los visitantes de las ciudades históricas españolas: características generales y rasgos específicos inducidos por la celebración de eventos turístico-culturales. En Ortega, E. (Coord.) Investigación y estrategias turísticas (pp.37-64) Madrid: Thomson.

Troitiño, M.A.; De la Calle, M.; García, M. y Troitiño, L. (2009). Observatorio Turístico del Grupo de Ciudades Patrimonio de la Humanidad de España. Informe 2008. Madrid: Universidad Complutense de Madrid. 
Tyrrell, T. and Johnston, R. (2001).A framework for assessing direct economic impacts of tourism events: Distinguishing origins, destinations, and causes of expenditures. Journal of Travel Research, 40(1), 94-100.

Valdés, L. Aza, R., y Baños, J. (2010). Impacto económico del turismo en una región: el caso de Asturias. Cuadernos de Economía, 33 (91), 183-208

Valdés, L., Del Valle, E. y Sustacha, I. (2011). El conocimiento del turismo en el ámbito regional. Cuadernos de Turismo, 27,931-952.

Váldes, L., Martín, R. y Aza, R. (2011). Diseño del sistema de información turística para la Habana. El SITCHA, implementación adaptada del Sistema de Información Turística de Asturias (SITA). Estudios Turísticos, 189, 151-171.

Villafranca, M.M. y Chamorro, V.E. (Eds.) (2007). Estudio de impacto económico del Conjunto Monumental de la Alhambra y Generalife en la Ciudad de Granada. Granada: Consejería de Cultura de la Junta de Andalucía y Comares Ediciones.

\section{Páginas Web}

ANCMPM (2015). Asociación Nacional de Ciudades Mexicanas del Patrimonio Mundial. $\begin{array}{llllll}\text { Visitado el } & 26 & \text { de enero de } & 2015\end{array}$ http://ciudadespatrimonio.mx/ancmpm/observatorio-turistico/

Ayuntamiento de Ávila (2014). Boletín Informativo del Observatorio Turístico de la Ciudad de Ávila. Visitado el 19 de diciembre 2014 en http://www.avilaturismo.com/es/areaprofesional/boletin-tco

Observatorio de Santiago de Compostela (2014). Visitado el 19 de diciembre de 2014 en http://www.usc.es/gl/institutos/cetur

Observatorio Turístico de Colonia (2014). Visitado el 7 de diciembre de 2014 en http://coloniaturismo.com/index.php?option=com k2\&view=item\&id=217:observat orio-tur\%C3\%ADstico-de-colonia\&Itemid=523\&lang=es

Observatorio Turístico del Estado de Guanajuato (2014). Visitado el 13 de diciembre de 2014 en http://www.observatorioturistico.org/

OCPM (2013). Listado de ciudades por región. Visitado el 16 de noviembre de 2013 en http://www.ovpm.org/en/cities/by region

SECTUR (2015). Observatorios Turísticos. Secretaría de Turismo de México. Visitado el 26 de enero de 2015 en http://www.ricit.sectur.gob.mx/es es/RICIT/Directorio de Observatorios Turisticos

SIET (2015). Sistema de Información y Estadística Turística de Argentina. Visitado el 26 de enero de 2015 en http://siet.desarrolloturistico.gov.ar/

Turismo de Córdoba (2014). Visitado el 19 de diciembre de 2014 en http://www.turismodecordoba.org/observatorio-turistico.cfm

Turismo de Salamanca (2014). Visitado el 19 de diciembre de 2014 en http://www.salamanca.es/es/profesionales/item/210-observatorio-turistico

Turismo de Segovia (2014). Visitado el 19 de diciembre de 2014 en http://www.turismodesegovia.com/es/prensa/observatorio-turistico

\section{ANEXO}

\section{Guía para el estudio de los observatorios turísticos de ciudades patrimoniales}

\section{Sección 1. Datos generales}


1. ¿Desde qué fecha funciona el observatorio?

2. ¿A qué entidad se subordina?

3. ¿El observatorio está integrado por agentes públicos y/o privados?

4. ¿El observatorio cuenta con una página web?

\section{Sección 2. Recopilación, procesamiento y difusión de la información}

5. ¿El observatorio utiliza fuentes de informaciones primarias y secundarias? -

6. ¿Se realizan estudios de la demanda en destino y en origen?

7. ¿Se realizan estudios de la oferta?

8. ¿Se realizan estudios sobre el impacto económico del turismo?

9. ¿Se realizan estudios sub-sectoriales o de productos turísticos específicos?

10. ¿Se realizan estudios sobre la población local?

11. ¿Existe una metodología de tratamiento de la información?

12. La difusión de la información se realiza a través de: Página web Informes publicaciones específicas

13. ¿Quién o quiénes son los principales destinatarios de la información?

14. ¿En qué proyectos de investigación relacionados con la ordenación de la actividad turística en el Centro Histórico ha colaborado?

\section{Sección 3. Redes de cooperación y TIC}

15. ¿El observatorio pertenece a alguna red o grupo de trabajo? ¿Éste cuenta con una página web? ¿Qué publicaciones se realizan?

16. ¿Cómo se emplean las Tecnologías de la Información y la Comunicación en beneficio de la recopilación de datos de los visitantes? 